\title{
Current trends in aortic intramural hematoma management- a shift from conservative to a more aggressive treatment
}

\author{
Konstantinos Spanos ${ }^{1,2}$, Tilo Kölbel ${ }^{2}$, Athanasios D. Giannoukas ${ }^{1}$ \\ ${ }^{1}$ Department of Vascular Surgery, University Hospital of Larissa, Faculty of Medicine, School of Health Sciences, University of Thessaly, Larissa, \\ Greece; ${ }^{2}$ Department of Vascular Medicine, German Aortic Center, University Heart Center, Hamburg, Germany \\ Correspondence to: Konstantinos Spanos, MD, MSc, PhD. Department of Vascular Medicine, German Aortic Center Hamburg, University Heart \\ Center, University Hospital Hamburg Eppendorf Martinistr. 52, 20246 Hamburg, Germany. Email: spanos.kon@gmail.com.
}

Submitted Apr 20, 2019. Accepted for publication May 31, 2019.

doi: $10.21037 /$ acs.2019.06.02

View this article at: http://dx.doi.org/10.21037/acs.2019.06.02

An aortic intramural hematoma (IMH) is defined as the presence of blood within the aortic wall without intimal disruption or an identifiable entry point on imaging. IMHs are either a consequence of the rupture of the vasa vasorum in the medial layer of the aortic wall, or the outcome of an intimal entry tear, allowing blood from the aortic lumen to enter the aortic wall and thrombosing due to stasis within the intimal layer (1). The incidence of IMH ranges from $5-25 \%$ of acute aortic syndromes and it is categorized similarly to aortic dissection, as either Stanford type A or B. Stanford type A IMH is associated with higher rates of morbidity (e.g., pericardial/pleural effusion, progression to dissection, aneurysm formation, rupture), and mortality than Stanford type B IMH (2). Computed tomography angiography signs can identify high-risk patients with IMH prone to higher morbidity and mortality: IMH with maximum ascending aortic diameter from 48 to $55 \mathrm{~mm}$ or descending aortic diameter $>41 \mathrm{~mm}$ was predictive of adverse events. Thickness hematoma $>11 \mathrm{~mm}$ is also predictive of adverse events-independent of the maximum aortic diameter. Greater ulcer-like projection diameters and depths have been associated with an increased risk of complications, with cutoff points in diameter of 10-20 mm and depth of 5-10 mm (Figure 1) (2).

Although IMH seems to have a better prognosis than aortic dissection, patients with IMH are at significant risk for progressive aortic-related morbidity, including progression to dissection, aortic rupture, aneurysm formation, and ulceration. Surgical treatment of an aortic IMH involving the ascending aorta with open replacement of ascending aorta results in lower mortality and longer survival compared to conservative management. Recently, Hata et al. (3) showed that emergency surgery for patients with type A IMH showed favorable mortality rates because most of the patients were in a hemodynamically stable condition preoperatively. On the other hand, several patients died suddenly during conservative treatment. Matsushita et al. (4) highlighted that patients with type A IMH might be different patients from those with aortic dissection, with different preoperative clinical characteristics. Thus, IMH patients were older, included a higher ratio of female patients and more frequently had hypertension, hyperlipidemia and cardiac tamponade. In this cohort of patients, emergency open surgery for type A IMH demonstrated low operative mortality and excellent 5-year survival. Open type A IMH repair seems to have good results, although, IMH patients may also be offered endovascular repair. Recently, a systematic review of the literature highlighted that despite the absence of a dedicated aortic stent-graft for the ascending aorta, patients with a spectrum of ascending aortic diseases are being successfully treated by endovascular technologies (5).

An IMH affecting the descending aorta can be managed with medical or with endovascular approach (6). The recent European Society for Vascular Surgery (ESVS) guidelines recommend that endovascular repair (TEVAR) should be considered for complicated type B IMH (IIa, C). Bischoff et al. (7) demonstrated that although conservative treatment is justified in uncomplicated type B IMH, it becomes complicated in the majority of patients within the first 20 days and thus an intervention may be necessary as it is a feasible option. Recently, Piffaretti et al. (8) highlighted 

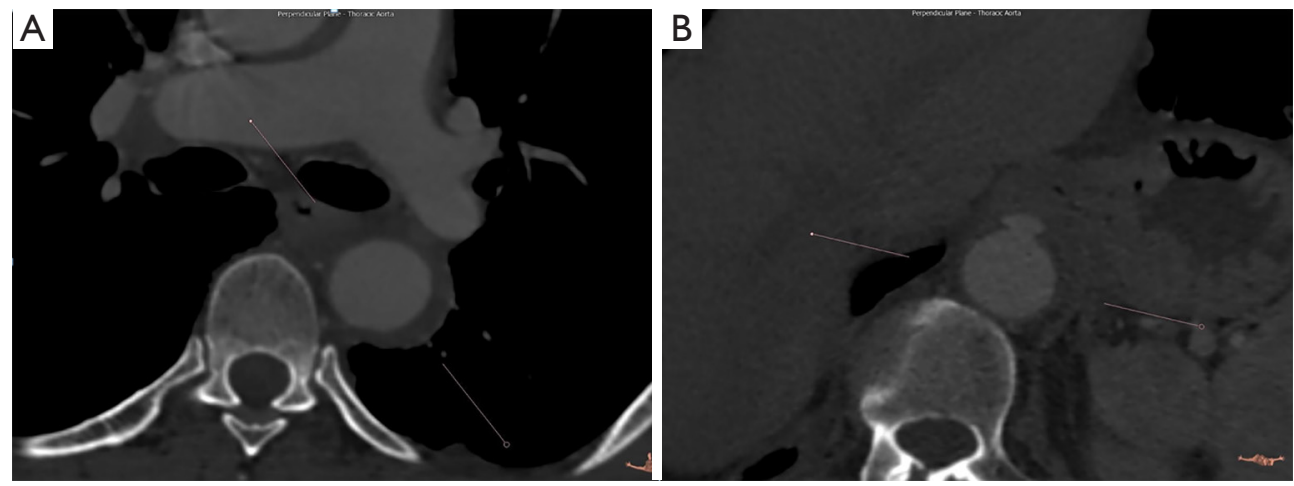

Figure 1 The thickness of hematoma $>11 \mathrm{~mm}$ is predictive of adverse events, independent of the maximum aortic diameter. Greater ulcerlike projection diameters and depths have been associated with an increased risk of complications, with cutoff points in diameter of 10-20 mm and depth of 5-10 mm. (A) Thickness of hematoma; (B) greater ulcer-like projection diameter and depth.

that type B IMH was associated with $47 \%$ aortic adverse event rate in the follow-up with conservative treatment alone, while the mid-term outcomes of TEVAR were more encouraging. The remodeling of the aorta after type B $\mathrm{IMH}$ is an important issue. Lavingia et al. (9) reported that TEVAR is not only safe and effective in treating IMH, but also based on longitudinal computed tomography scan analysis, aortic remodeling was evidenced by normalization of all measured anatomical indices. Along these lines, it was recently suggested that occurrence of complete aortic remodeling was significantly lower in the conservative group, while TEVAR is likely to protect from progression of type B IMH to dissection and thus, it may be associated with a better prognosis (15.4\% vs. $82.1 \%$ in TEVAR group, $\mathrm{P}<0.001)(10)$.

Unresolved issues regarding the treatment- particularly endovascular approaches-of IMH still exist. Thus, endovascular techniques and devices are still under assessment for the treatment of ascending aorta IMHs, with patient-specific anatomical differences playing a part in their suitability for TEVAR. There is also a potential risk of induced entry points and aortic dissection during or after endovascular intervention. Cost-effectiveness is another issue; the interventional cost including the device price, frequent imaging examination costs-particularly in conservative management-with radiation exposure and contrast, plus the potential cost of an intervention in those patients who were initially treated conservatively. Experienced centers have already applied endovascular techniques to the ascending aorta and arch pathologies with encouraging outcomes $(5,11)$. Future studies should assess the efficacy of endovascular treatment of both type A and $\mathrm{B}$ IMH in order to provide more robust evidence for the management of IMH.

\section{Acknowledgments}

None.

\section{Footnote}

Conflicts of Interest: T Kölbel is an intellectual property holder in Cook Medical and he is a proctor for Cook. The other authors have no conflicts of interest to declare.

\section{References}

1. Riambau V, Böckler D, Brunkwall J, et al. Editor's Choice - Management of Descending Thoracic Aorta Diseases: Clinical Practice Guidelines of the European Society for Vascular Surgery (ESVS). Eur J Vasc Endovasc Surg 2017;53:4-52.

2. Kruse MJ, Johnson PT, Fishman EK, et al. Aortic intramural hematoma: Review of high-risk imaging Features. J Cardiovasc Comput Tomogr 2013;7:267-72.

3. Hata $M$, Hata H, Sezai A, et al. Optimal treatment strategy for type A acute aortic dissection with intramural hematoma. J Thorac Cardiovasc Surg 2014;147:307-11.

4. Matsushita A, Fukui T, Tabata M, et al. Preoperative characteristics and surgical outcomes of acute intramural hematoma involving the ascending aorta: A propensity score-matched analysis. J Thorac Cardiovasc Surg 
2016;151:351-8.

5. Muetterties CE, Menon R, Wheatley GH 3rd. A systematic review of primary endovascular repair of the ascending aorta. J Vasc Surg 2018;67:332-42.

6. Attia R, Young C, Fallouh HB, et al. In patients with acute aortic intramural haematoma is open surgical repair superior to conservative management? Interact Cardiovasc Thorac Surg 2009;9:868-71.

7. Bischoff MS, Meisenbacher K, Wehrmeister M, et al. Treatment indications for and outcome of endovascular repair of type B intramural aortic hematoma. J Vasc Surg 2016;64:1569-79.e2.

8. Piffaretti G, Lomazzi C, Benedetto F, et al. Best Medical Treatment and Selective Stent-GraftRepair for Acute Type
B Aortic Intramural Hematoma. Semin Thorac Cardiovasc Surg 2018;30:279-87.

9. Lavingia KS, Ahanchi SS, Redlinger RE, et al. Aortic remodeling after thoracic endovascular aortic repair for intramural hematoma. J Vasc Surg 2014;60:929-35; discussion 935-6.

10. Ye K, Qin J, Yin M, et al. Acute Intramural Hematoma of the Descending Aorta Treated with Stent Graft Repair Is Associated with a Better Prognosis. J Vasc Interv Radiol 2017;28:1446-53.e2.

11. Tsilimparis N, Detter C, Law Y, et al. Single-center experience with an inner branched arch endograft. J Vasc Surg 2019;69:977-85.e1.

Cite this article as: Spanos K, Kölbel T, Giannoukas AD. Current trends in aortic intramural hematoma managementa shift from conservative to a more aggressive treatment. Ann Cardiothorac Surg 2019;8(4):497-499. doi: 10.21037/ acs.2019.06.02 